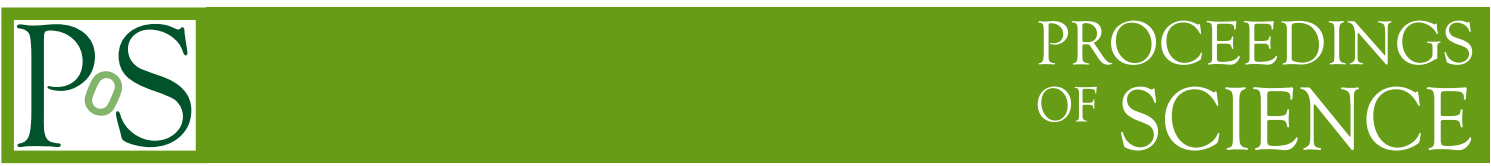

\title{
An Update on TRAPUM
}

\author{
Ben Stappers* \\ Jodrell Bank Centre for Astrophysics, School of Physics and Astronomy, University of \\ Manchester \\ E-mail: Ben.Stappers@manchester.ac.uk

\section{Michael Kramer} \\ Max-Planck-Institut für Radioastronomie, Auf dem Hügel 69, D-53121 Bonn, Germany \\ Jodrell Bank Centre for Astrophysics, School of Physics and Astronomy, University of \\ Manchester \\ E-mail: michael@mpifr-bonn.mpg · de
}

\begin{abstract}
TRansients and PUlsars with MeerKAT (TRAPUM) is a MeerKAT Large Survey Project which will utilise MeerKAT to greatly expand our knowledge of the populations of sources which emit at radio wavelengths on timescales ranging from microseconds to seconds. The excellent sensitivity of MeerKAT will allow us to discover many new (millisecond) pulsars, fast radio transients including FRBs, radio emitting magnetars, RRATs, giant pulses, flare stars, and active binaries to name just a few. It will also allow us to significantly expand the parameter space over which radio transients have been discovered. MeerKAT will also be excellent for localising and new sources through the combination of the relatively long baselines and the use of transient buffers. TRAPUM will achieve this through a combination of targeted searches: directed at gamma-ray emitters identified by Fermi, supernova remnants and other high energy sources, globular clusters - reservoirs of pulsars, and the Galactic centre, and blind searches of regions of the Galactic plane. It will also perform a wide area Fly's Eye style search for fast transients that will be able to find at least 30 of the brightest FRBs. These searches are enabled by MeerKAT in combination with a 400-beam beamformer and real-time and offline processing capabilities funded through the Max-Planck-Institut für Radioastronomie and the European Research Council-funded MeerTRAP project. This contribution is completed on behalf of the entire TRAPUM team which consists of more than 40 members from across the world. See www.trapum.org for up to date team membership and other information.
\end{abstract}

MeerKAT Science: On the Pathway to the SKA

25-27 May, 2016

Stellenbosch, South Africa

${ }^{*}$ Speaker. 


\section{Science goals of TRAPUM}

The scientific aims of the TRAPUM project are to greatly expand our knowledge of the radio sky on timescales ranging from microseconds to seconds and thereby achieve the following goals.

- Discover exotic systems containing pulsars and compact companions and new millisecond pulsars to enable improved tests of general relativity and the search for gravitational waves.

- Discover pulsars in the Galactic centre and use them to probe gravitational effects of Sgr A*, dark matter and stellar populations.

- Expand number of transient radio sources: RRATs, giant pulses, flare stars, active binaries.

- Greatly expand the parameter space: sensitivity; search volume; time resolution, search for fast radio transients to study the highest energy density events in the Universe; and where possible use them for cosmology, or gravitational wave emission studies.

- Increase the populations of all neutron stars to constrain birth rates, spatial and velocity distributions, to understand the number of neutron stars, types and formation rates.

- Study the properties, dynamics, and evolution of globular clusters (GCs) by finding new pulsars, including exotic systems which can be used to determine neutron star masses.

- Investigate the dependence of the pulsar and fast transient populations on properties such as metallicity and star formation history, by searching for them in external galaxies

We now describe the motivation and goals of the TRAPUM project in more detail.

\subsection{Targeted pulsar searches of SNRs, PWNe, and unidentified Fermi gamma-ray sources:}

Supernova remnants (SNRs), pulsar wind nebulae (PWNe) and Fermi gamma-ray sources host, arguably, some of the most interesting radio pulsars. The discovery of a radio pulsar coincident with a SNR/PWN/gamma-ray source is crucial for understanding the energy budget of such systems and, vice-versa, multi-wavelength counterparts provide substantially more context for understanding the nature of the radio pulsar itself. Discovering young pulsars associated with SNRs or PWNe is important for understanding the Galactic neutron star formation rate, the nature of the supernova explosion, and the injection of high-energy particles into the interstellar medium. Unidentified Fermi gamma-ray sources provide a treasure map for deep pulsar searches and, for example, the MSPs found can probe accretion physics (e.g. "transitional" MSPs), provide new precision timers for the International Pulsar Timing Array, as well as identify exotic binaries capable of testing gravity and/or constraining the neutron star equation of state. With a 400-beam pulsar search mode, MeerKAT is poised to revolutionise pulsar searches of SNRs/PWNe/gamma-ray sources in the Southern sky $\left(\sim \delta<-39^{\circ}\right)$.

Since its launch in 2008, Fermi has revolutionised our view of the gamma-ray sky. Over 200 gamma-ray pulsars are now known, roughly half of which are MSPs [1]. Radio pulsar timing and deep radio pulsation searches have been indispensable to the success of Fermi in this area. Over 70 MSPs have been discovered in targeted searches of Fermi gamma-ray sources. Excitingly, this 
is just the tip of the iceberg: there remain $\sim 1000$ unidentified Fermi gamma-ray sources, a large fraction of which are likely to be pulsars [2]. Through TRAPUM, MeerKAT can play a major role in solving the lingering mystery of the nature of these sources and produce a large yield of pulsar discoveries to address the scientific goals mentioned here. A recent classification of the Fermi 3FGL catalog unassociated sources [2] provides us with a new list of 334 potential pulsar targets inferred from $\mathrm{GeV}$ measurements, of which 125 are below $\delta=-39^{\circ}$. Targeting these sources as part of TRAPUM, which can reach a sensitivity $5-10 \times$ deeper than the Parkes radio telescope - the next best option for searching such southern sources. MeerKAT's sensitivity will be crucial because it is likely that many of these sources will be very faint $(<100 \mu \mathrm{Jy})$.

Considering SNRs and PWNe, we note that many still have no associated young pulsar, which remains a mystery and greatly limits our ability to understand their energetics, formation history, and the statistics of the population. TeV gamma-ray observations with HESS have transformed our view of PWNe - in fact, PWNe are the predominant Galactic source class of $\mathrm{TeV}$ emitters and are sometimes associated with young, faint radio pulsars (e.g., [3]). Moreover, the Cherenkov Telescope Array (CTA) will go an order of magnitude deeper and unveil a large population of SNRs, PWNe and gamma-ray binaries in the TeV domain. To our current knowledge, there are more than 250 SNRs and PWNe without any known associated pulsar and visible from the Karoo (from the Green 2014 catalog and HESS Galactic plane survey), the vast majority of which have sky extensions comparable to the TRAPUM 400-beam FOV ( $\sim 14$ arcmin), thus requiring only a single pointing. We aim to deeply search all of these.

Searches of unidentified Fermi gamma-ray sources will require 54 min integrations to reach a $10 \mu \mathrm{Jy}$ pulsar $\left(S_{1400}\right)$. As shown in [4], who used three passes per source, multiple visits are critical for finding eclipsing ${ }^{1}$ and/or highly accelerated MSPs. In fact, pulsars have undoubtedly been missed in previous searches for all of the aforementioned reasons. Following the same strategy, and based on previous search results, we estimate that we will discover a pulsar in one-fifth of the gamma-ray sources we search (i.e. $\sim 25$ new pulsars). In total, 338 hours for searching would be needed for the 125 targets below $\delta=-39^{\circ}$.

SNR/PWN searches for young pulsars will require typically 9 min per target for a $40 \mu \mathrm{Jy}$ pulsar assuming a $10 \mathrm{~K}$ excess of sky temperature in each of the individual pencil beams due to the flux density contribution from the background nebula. ${ }^{2}$ In this case only two passes would be required in order to automatically confirm weak candidate pulsars and to start building a rotational ephemeris for the discoveries. Again assuming a one-fifth success rate this results in 50 new pulsars and the time required to search the 250 targets is $92 \mathrm{~h}$. The total time required for such targeted searches is thus $338+92=430 \mathrm{~h}$.

\subsection{Globular Cluster Searches}

Globular clusters (GCs) harbor a very large number of MSPs per unit stellar mass compared with the Galactic plane (see [5] for a recent review). This is because the dense stellar environments in the cores $\left(10^{4}-10^{3} \mathrm{M}_{\odot} \mathrm{pc}^{-3}\right)$ promote collisions and exchange interactions that create binaries

\footnotetext{
${ }^{1}$ We expect many of these sources will be redbacks and black widow systems.

${ }^{2}$ For sources with less background temperature, it would be useful to aim deeper to reach $20 \mu \mathrm{Jy}$, being equivalent with the flux of the weakest youngest pulsar known. The small MeerKAT beam is an advantage here.
} 
capable of recycling old neutron stars to become MSPs. A total of 146 pulsars have been discovered in globular clusters to date ${ }^{3}$, the majority of them MSPs. Some clusters are spectacularly prolific: Terzan 5 and 47 Tuc host 34 and 25 pulsars respectively. Surveying them with MeerKAT therefore has the potential for rich and rapid reward.

GCs are typically very distant objects and combined with the intrinsic faintness of most MSPs, implies that the vast majority of the pulsar population in GCs (between 600 to 3700; [6]) remains undetected, despite the depth of current surveys. Increasing the depth through longer integrations is not practical, because any objects found would be too faint for follow up studies. Progress can only be made with more sensitive telescopes as illustrated by the step change in the number of GC pulsars known coinciding with the availability of the GBT, finding 71 new MSPs (e.g., [7]).

MeerKAT has a similar discovery potential. Its Southern location and large collecting area will provide a leap in sensitivity in a large area of the sky inaccessible to the GBT and only accessible to the Parkes telescope. MeerKAT is about 6 times more sensitive than the most recent Parkes surveys of GCs and similarly sensitive to the GBT, and therefore has the potential for a similarly rich return as the GBT. We prioritise GCs with high masses and large interaction rates, where more MSPs should form (47 Tuc (NGC 104), Omega Centauri (NGC 5139), NGC6388, and Liller1). The latter two systems have been searched with the GBT, but MeerKAT can go two times deeper in sensitivity by utilising a longer integration time. Another reason to target the aforementioned clusters is that they have been detected in gamma-rays with Fermi. This emission is very likely due to the integrated gamma-ray emission from their MSP populations. Other clusters we propose to search that have strong gamma-ray emission (and a likely large number of MSPs) are 2MS-GC01, NGC2808, NGC 6093 (M80), NGC6361, NGC6541, NGC6652 and NGC6717.

These GCs span a range of dynamical states, from low-density cores like Omega Centauri to core collapsed systems and this affects the pulsar populations to be found in each cluster. Clusters with very dense cores have much higher rates of interaction per binary (e.g. [8]) As a consequence binaries can be disrupted and we get partially recycled pulsars. The pulsars we will find in these GCs will be crucial to test this hypothesis. The core collapse clusters also have many "secondary" exchange products: normal recycled pulsars where the low-mass white dwarf companion was exchanged for another star, forming an "exotic" system. Sometimes this other star is a massive white dwarf or another neutron star, invariably in highly eccentric orbits. Until now, the only two systems for which we have multiple post-Keplerian effects that allow precise NS mass measurements (NGC 6544B, [9]) are well-known secondary exchange binaries produced in two core-collapsed clusters. Two other clusters with these types of exotic binaries that we propose to observe more deeply are NGC 1851 [10] and NGC 6652 [11]. We also plan to observe the following core-collapsed clusters that are too far south for the GBT, namely NGC 6397, NGC 6752, NGC 362, NGC 5946, as they have high potential for finding new, exotic binaries and might include a double MSP or a pulsar-black hole system, which would be superb for testing gravity theories.

Two long observing tracks on each of these 16 clusters taken at different times to compensate for sensitivity losses due to eclipses, high orbital accelerations, and unfortunate scintillation will reveal the majority of the pulsars in these clusters. For all the clusters full tracks (from rise to set) would correspond to an average of 10-h per cluster, giving us the maximum sensitivity that can be

\footnotetext{
${ }^{3}$ See http://www.naic.edu/\%7pfreire/GCpsr.html and references therein.
} 
realised with MeerKAT compared to previous surveys. Compared to the recent Parkes surveys, this results in the improvement in the sensitivity by a factor of 6 . Given the luminosity law for pulsars in GCs (e.g., [12]), this should result in a factor of 6 improvement in the number of pulsars detected. For 47 Tuc, this implies the discovery of more than 100 new pulsars; however, many faint pulsars have been discovered already there (because of scintillation and the sheer number of observations already made), so the number of new pulsars will be of the order of 10 . For the remaining clusters in the Parkes-only sky, the number of known pulsars is 6 (5 in NGC 6752 and 1 in NGC 6397). A factor of 6 improvement implies about 30 new pulsars. For the remaining clusters without known pulsars, these will enable the first discoveries. For clusters visible with the GBT, the improvements are harder to calculate because each cluster has been surveyed to a different depth, depending on its declination. The Southern-most GC targeted by the GBT was NGC 6388, observed only for two hours. Here, a 10-hour track results in almost twice the sensitivity. Using the average rise- to set-time, and the two tracks, a total of $320 \mathrm{~h}$ would be required.

\subsection{Extragalactic pulsar and transient searches}

MeerKAT has the sensitivity to reveal new pulsars and fast transients beyond the Milky Way. Using these systems we can determine the relationship between metallicity, most recent epoch of star formation and their formation. Only 29 pulsars are known in the Magellanic Clouds (6 in the SMC and 23 in the LMC). Using MeerKAT it will be possible to reach a minimum detectable flux density of $\sim 7 \mu \mathrm{Jy}$ in 2 hours for periodic sources, and a limit of $\sim 50 \mathrm{mJy}$ for 1 -ms duration individual pulses. This would result in a flux limit about a factor of 7 more sensitive than the deepest Parkes observations [13]. We have identified many regions of interest in the LMC/SMC based on the location of known pulsars, X-ray binaries, star formation regions and supernova remnants. Our analysis shows that their distribution is such that we can observe the majority of them using a limited number of pointings of the 400 tied-array beams. Using the incoherent beams in parallel the bulk of both galaxies can also be tiled out simultaneously. Our calculations show that we need 8 and 26 pointings for the SMC and LMC, respectively, in order to achieve the required spatial coverage. A total of 136 hours for 34 pointings, each of two hours duration and each observed twice so that the above flux limit and possible variability can be probed will be required.

Current models for large-scale structure formation predict that matter in the current epoch forms a "cosmic web". Most of the baryons in the Universe reside in large-scale filaments that form the "strands" of the web, with groups and clusters of galaxies located at the intersections of filaments. Detecting pulsars and fast transients outside the local group, and determining how much their signal was dispersed by the intergalactic medium will begin to provide us with the tools needed to probe this structure. Understanding the nearby population of giant pulse emitting, or radio-emitting magnetars, has gained even more importance given that they are proposed models for at least some of the FRBs and this is further highlighted by the recent discovery of a repeating FRB [14]. We have chosen 10 sources at the edge of, and beyond, the local group, where we are sensitive to any Crab-like giant pulses. These hosts range from e.g. IC 1613 (0.7 Mpc) to NGC 7793 (3 Mpc); while Fornax, at a distance of $17 \mathrm{Mpc}$, allows us to simultaneously view a large number of galaxies, and a chance to probe a significant fraction of the IGM. Comparison with the best limits, either from Arecibo [15] or the HTRU survey [16] we find that TRAPUM will be able to go at least a factor of 6 deeper, and have up to an order of magnitude more observing time, greatly 
enhancing the chance of detecting giant pulses. We therefore request time for NGC 253, NGC 300, Fornax, NGC 6300, NGC 7793, Sextans A, Sextans B, NGC 3109, IC 1613 and IC 342, of 3-hr integrations using both the coherent and incoherent beams, repeated thrice to allow for variability. Overall this results in a total observing time of $10 \times 3+136=226 \mathrm{~h}$.

\subsection{Using pulsars to probe gravity, dark matter \& stellar populations in the Galactic Centre}

The discovery of a pulsar closely orbiting the super-massive black hole at the centre of our Galaxy, Sgr A*, would not only supersede all previous tests of General Relativity (GR) in the strong-field regime [17, 18], it would also enable the space-time around a rotating black hole to be probed with high precision and in a model independent fashion; for example, allowing tests of the cosmic censorship conjecture and the no hair theorem $[19,20]$. Such a "laboratory" for precision tests of GR and black hole physics would be unrivalled by any future astrometric measurements of the S-Stars [21]. Furthermore, mass-segregation in the central parsec may also lead to the presence of additional gravitational testbeds in the form of stellar-mass pulsar black hole binaries [22].

In addition to using pulsar orbits about Sgr A* for high precision measurements of the central black hole's properties (e.g. [23]), each pulsar allows us to probe the turbulent magnetized interstellar medium in the extreme environment around the black hole. This is possible through measurements of pulse dispersion, scattering and Faraday rotation as demonstrated by the recent discovery of a radio-loud magnetar within $0.1 \mathrm{pc}$ of Sgr A* [24]. Remarkably, pulsars in the Galactic centre $(\mathrm{GalC})$ also offer the possibility of tests of physics beyond the standard model: recently, data from the Fermi have revealed an "excess" in the diffuse emission at $\mathrm{GeV}$ energies (1-3 GeV) on degree scales towards the GalC [25]. One explanation has been annihilating Dark Matter in the Galactic nucleus, however, the observed spectrum is also fitted well by a large population (thousands) of unresolved MSPs, perhaps originating from disrupted Globular Clusters [26]. MSPs that emit in gamma-rays have also been shown to be radio emitters, and as such targeted radio surveys of the Galactic bulge are a tantalising prospect to test this hypothesis. Pulsars close to the black hole can hence be used to limit Dark Matter models [27]. Previous GalC surveys were insensitive to MSPs [28] and were focused on the very inner few degrees. However, supported by the diffuse gamma-ray emission, we expect the bulge MSP population to extend to comparatively high latitudes of 5 to 10 degrees. We estimate that the bulge population contains $(2.7 \pm 0.9) \times 10^{3}$ radio-bright $\left(S_{1400}>10 \mu \mathrm{Jy}\right) \mathrm{MSPs}$ [29].

In line with the goals defined in 2010 , the aim is (a) to find pulsars in close proximity of Sgr A* to probe the black hole properties, and (b) to understand both the GalC population by clearly identifying and characterising the central and bulge population of MSPs, also establishing a robust link to the Fermi $\mathrm{GeV}$ excess. We would search a region with diameter of $1 \mathrm{deg}(\sim$ $150 \mathrm{pc}$ ) centered on $\mathrm{Sgr} \mathrm{A}^{*}$ with special emphasis on the inner arcminute centered on Sgr A*. Our observing strategies accommodate updated knowledge about scattering from electron-density variations [30], which causes pulse broadening that reduces the sensitivity to both pulsars and transients. Pulse broadening is highly frequency dependent $\left(\tau \propto v^{-4}\right)$ and varies both across the GalC region and as a function of radial depth through the region. For this reason it would be better to search at different frequencies. (i) Survey of the GalC with the MPIfR S-band system, where about 7 pointings are needed to cover the region. A dwell time of $6 \mathrm{hr} /$ pointing allows sampling of about $1 / 2$ the luminosity function for the longest period objects where scattering is 
less of an issue. We will conduct this part of the survey within the time dedicated to the MPIfR S-band survey. (ii) Deep survey around Sgr $\mathrm{A}^{*}$ at multiple frequencies with multiple passes to contend with spin-axis (geodetic) precession, eclipses, and intrinsic intermittency. In addition to S-Band it would be desirable to observe 10 epochs each at X-band (three pointings each) to sample comparable luminosity. This would result in an additional $120 \mathrm{~h}$ and $180 \mathrm{~h}$, respectively. We are aware that the required receivers are not available yet and that this project would not be possible until they are. (iii) Galactic Bulge survey at L-band to test GalC Dark Matter hypothesis. Using our simulations [29] where we assume that the spatial distribution of bulge MSPs traces the Fermi LAT GeV excess we determined the number of bulge and disk MSPs in $4 \mathrm{deg}^{2}$ regions, that can be found in 30 minute observations with TRAPUM, around the Galactic centre. We find that the optimal search regions to extract the bulge MSP population are a few degrees north and south of the GalC, at approximately $-3^{\circ} \leq|\ell| \leq 3^{\circ}$ and $5^{\circ} \leq|b| \leq 9^{\circ}$. We find that the difference in the expected Dark Matter distribution of detectable bulge and thick-disk MSPs is rather large, such that the detections of just $\sim 5$ bulge sources suffices to establish the detection of the bulge population at the $3 \sigma$ level. However, in order to fully establish whether the Fermi GeV excess is caused by the bulge MSP population, it will be necessary to estimate the density and distribution of bulge MSPs to high enough accuracy. Aiming at a statistical error of $\sim 20 \%$, this requires the detection of about $\sim 25$ bulge MSPs (and $\sim 18$ disk MSPs). This is possible with $300 \mathrm{~h}$ of survey time in our optimally chosen regions. We note that this area is highly complementary to that of the plane survey described below.

\subsection{Towards a Galactic census}

Since the first plans for TRAPUM proposed in 2010, the known pulsar population has increased by about 400 sources, or about $20 \%$. This increase has been achieved by improving techniques and methods on existing telescopes, and new telescopes like LOFAR. With new pulsars, new science is enabled, resulting from the bulk properties of the discovered population, from increasing the number pulsars that are probes of the surrounding medium, or those that are exceptional laboratories for testing theories of gravity. As MeerKAT is many times more sensitive than Parkes, the previous largest dish used for pulsar searches in the South, the search for pulsars in the Galactic plane - the birth place of pulsars - provides a significant and rare sharp increase in sensitivity for exploring the dynamic radio sky. The 400 beams combined with much increased sensitivity mean a significant increase in search capability, making a large-scale survey with MeerKAT not only possible, but in fact mandatory. A TRAPUM Galactic Plane survey will be the most sensitive survey of the inner Galactic plane ever conducted and would be the benchmark and testbed for the later SKA surveys.

The most successful survey to date, the Parkes Multibeam Survey (PMPS), combined with its follow-up surveys HTRU and SUPERB [16] covered a longitude range from $l=-80$ deg to $l=+30$ deg with a latitude range of $|b| \leq 5 \mathrm{deg}$. We have conducted simulations to gauge the most efficient yield in terms of new discoveries per covered area, based on the PSRPOPpy package [31]. Based on these, we find that a Galactic plane survey covering $-60 \mathrm{deg} \leq l \leq+30$ deg and $|b|<3$ $\mathrm{deg}$, resulting in a survey area of $540 \mathrm{deg}^{2}$, is the most promising. In contrast to the deepest survey in this area, HTRU-LowLat [32], which employed an integration of $4320 \mathrm{~s}$, the greater sensitivity of MeerKAT allows us to reduce this to $900 \mathrm{~s}$. With the larger bandwidth available and somewhat 
lower centre frequency (1250 MHz), the survey will be twice as sensitive as HTRU-LowLat, and up to 3.5 times more sensitive than PMPS. At the same time, the shorter integration time allows for searches of much more highly accelerated systems, i.e. those in tighter orbits and/or more massive companions. This is because the computational load scales strongly with observing time $\left(t_{o b s}^{3}\right)$ and so compared to HTRU-LowLat is reduced by factors of 100 and 20, respectively. ${ }^{4}$ Using our simulations, we predict about $600-800$ new pulsar discoveries. These numbers are naturally somewhat uncertain, but in particular the improved sensitivity to highly accelerated systems makes this survey tremendously promising for finding extreme compact binary systems, including the "holy grail" of a pulsar-black hole system, which will be a superb probe of gravity theories [20,23]. In order to cover the area requested, this part of the project would require 1350 hours.

\subsection{Fast transients - discovering and understanding source populations}

The fast transient landscape has changed dramatically since 2010 with the discovery of the population of FRBs [33] which are exciting in themselves but also highlighted that the dynamic radio sky is still largely unexplored and with potentially more rich rewards. MeerKAT's unique combination of wide FoV, high sensitivity, and wide bandwidth will provide supreme sensitivity per unit time and frequency making it a prime instrument to study the transient sky. We discuss some of the possible sources of transient emission below, but history has shown that the most exciting discoveries are the unexpected ones.

The field of fast radio transient detection was reignited with the discovery of 11 Rotating Radio Transients (RRATs, [34]). RRATs were shown to most likely be spinning neutron stars with periods of 0.1-8 s. As more RRATs are discovered the diversity in emission patterns argues that there is strong diversity in the class. Some appear to be extreme nullers and/or old pulsars close to the death line. While in others, a single pulse or a single short sequence of pulses is detected and the RRAT is never seen again [35]. These detections are consistent with cataclysmic events or a mechanism which generates rare conditions in an otherwise quiescent neutron star magnetosphere. Understanding the number of these objects and their relationship to the other neutron star manifestations is crucial for determining if there is a problem with the size of the Galactic neutron star population [36].

The fast transient parameter space was further expanded with the discovery of a highly dispersed millisecond transient in a $1.4 \mathrm{GHz}$ Parkes pulsar survey [37]. It was located out of the Galactic plane with a dispersion measure significantly exceeding the estimated contribution of Galactic ionised gas along the line of sight. With similar Parkes detections (see the FRB catalog and references therein), it became clear that highly-dispersed transients were a new class of radio source that are now called Fast Radio Bursts. FRBs detected at Arecibo [38] and the GBT [39] confirmed that these were not terrestrial and provided the first detections at frequencies outside the Parkes $1.4 \mathrm{GHz}$ bandpass.

The confirmation of FRBs as a class has led to many models of their progenitor (see [28] for summary) and ideas for their potential utility as probes of the ISM of other galaxies and the IGM. Recently FRBs have been detected at frequencies from $0.8-2 \mathrm{GHz}$ and one has been shown to repeat [14]. For this object, it rules out cataclysmic events: coalescing neutron stars [40], evapo-

\footnotetext{
${ }^{4}$ Note that a complementary survey of the inner Galaxy will be conducted as part of the "MPIfR S-Band Project".
} 
rating black holes [41], or collapsing supra-massive neutron stars (e.g. [42]). However, there may indeed be multiple classes of FRBs [43]. Constraints on scattering and scintillation have countered suggestions that Galactic flaring stars [44] or atmospheric phenomena [45] are responsible.

Accurately localising FRBs on the sky with the TRAPUM multi-beam searches enables their host galaxies to be unambiguously identified. The redshift can then be determined in the optical, which, in tandem with the precisely measured dispersion means new cosmology tests can be performed. The first of these is to 'weigh' the baryons in the IGM; the ionised component of these (including the so-called "missing baryons") is what causes the FRB's characteristic dispersion [43]. As the sample of FRBs detected in this way grows one should be able to 'see' clustering due to the strands of the cosmic web which will be traced on the sky in the FRB dispersion measure sky map for each redshift value. With a large sample of $\sim 100+$ FRBs the dispersion measure distribution as a function of redshift can be made. The shape of this distribution depends directly on the dark energy equation of state parameter. This gives a unique method (and orthogonal to efforts in other wavelength regimes) for measuring this parameter, and its redshift-dependent variation if any. More details can be found in [28].

On slow ( $>1$ second) time scales, radio transient studies have long focused on the rich science achievable with follow up of SNe and GRBs. GRBs are detected at rates of $\sim 600 \mathrm{yr}^{-1}$. If prompt radio emission is associated with these GRBs, and beamed like the $\gamma$-ray emission, MeerKAT will detect approximately one per year. The expectation is, however, that the radio emission is not beamed, allowing the detection of rapid transients associated with GRBs whose $\gamma$-ray emission is not detected. Radio-loud $\gamma$-ray quiet detections of these orphaned GRBs would allow the deduction of the true underlying rate.

The recent discovery of gravitational waves (GW) with LIGO provides radio astronomers with another incredible opportunity [46]. GW progenitors have potential electromagnetic counterparts with both fast coherent radio pulses and slow synchrotron afterglows. Their detection and crosscorrelation with events from GW detectors will not only shed light on the nature of such extreme events, but is likely the only way to localize GW events in order to locate any associated host galaxies. Some models of FRBs predict that coordinating with GW and optical afterglow networks would truly revolutionise our understanding of GWs, FRBs, the IGM, and much more.

Crucially compared to most other transient surveys, MeerKAT transient surveys using multiple tied-array beam modes will have resolution of a few arcseconds or better, sufficient to unambiguously associate events with sources in different wavebands. Arguably, MeerKAT has a smaller FoV than some other suitable pathfinders but as the full FoV is computationally challenging to explore, and as MeerKAT has a significantly higher sensitivity and can be used in coherent and incoherent modes, it is an outstanding instrument for the survey for transient sources. Furthermore, to find the rare bright events (such as Lorimer bursts or the prompt emission of GRBs) there are possibilities with fast imaging, sub-arraying, and forming incoherent sums and Fly's Eye modes. Our team has also already begun commissioning a modest "fast imaging" correlator mode that can perform imaging searches for transients over the entire field of view [47]. The Fly's Eye search (Alan Telescope Array; [48]) points each antenna in a different direction to incoherently search a large area of sky. We describe below three modes of operation.

Commensal-Transient-Searches-on-Pulsar-Searches Mode: We will carry out commensal observing for fast transients on all of the TRAPUM observations proposed here: Galactic plane and 
Centre, High energy point sources and SNRs, globular clusters and external galaxies. These offer rich and varied locations to search for all sorts of transients. In all cases we will use the incoherent sum of all 64 dishes to achieve a wide field of view, but lower sensitivity, to look for rare brighter sources, in parallel we will use the 400 tied-array beams to do narrower but much deeper searches. This combination allows for effective determination of luminosity relations with one survey. While there may be an apparent or real dearth of FRBs detected through the Galactic plane, the deep pulsar search provides an excellent and uniform way to determine whether this is true. It will also have sufficient sensitivity to be able to detect a few FRBs which help clarify if, and why, there is such a dearth. The Galactic plane pulsar search will also provide a rich region to search for Galactic fast transients such as RRATs, radio emitting magnetars, flare stars, active binaries, novae and any new classes of transients. The searches away from the plane discussed above provide excellent opportunities to find transients in those regions or in the Universe beyond. The follow up of known FRBs (see below) has already been successful in revealing a new FRB and here too the combination of on-sky time and sensitivity will reveal more sources. Similarly the follow up timing observations will add to on-sky time at a range of Galactic latitudes. The multiple visits planned for searches and follow-up timing will allow the opportunity to not only check the variability of new sources that are discovered, but also provide sensitivity to sources which show longer timescale variability, like the repeating FRB and some RRATs. The single pulse sensitivity is a factor of about 6 better than Parkes and the observing times will be significantly longer than the GBT and so we expect it will be possible to more than double the known RRAT population. Our simulations for the number of FRBs that we will detect in these commensal observations, based on the rate from [49], to be $10 \pm 5$ and $20 \pm 10$ bursts in the incoherent and tied-array modes respectively. This is a significant increase on the current known population and using our transient buffer and imaging capability they will all have positions of a few arcseconds or better based on the burst alone, an enormous improvement over the present situation.

Fly'e-Eye Mode: We consider here the exciting possibility of the detectability of Lorimer-burst brightness (30 Jy and $>400-\sigma$ ) FRBs with a MeerKAT Fly's Eye experiment. Comparing the differences in gain, $\mathrm{T}_{\text {sys }}$ and bandwidth of the single MeerKAT dish and Parkes we very conservatively assume that it is 15 times less sensitive. Thus a single MeerKAT antenna would have detected the Lorimer-burst at above $27 \sigma$. Based on the first discovery a rate of bright events of 225/sky/day was calculated, which agrees with the current FRB rates and the rarity of Lorimer-bursts among the new detections. In Fly's Eye mode, MeerKAT will instantaneously cover $0.8 \times 64=51.2 \mathrm{deg}^{2}$, or 0.00128 of the sky. An observing time of $720 \mathrm{~h}$ corresponds to 0.0384 sky days and the yield becomes $225 \times 0.0384 \sim 9$ Lorimer-bursts. Making the simplest assumption of a $\log N-\log S$ relation with an index of -1.5 , we estimate a total detectable FRB rate (above a conservative $\sim 13 \sigma$ ) of $\sim 1 \mathrm{FRB} /$ day, and a total yield of $\sim 30$ new detections in $720 \mathrm{~h}$. Discovering these bright bursts provides crucial information on the luminosity distribution and potentially very useful probes of the local IGM and the host galaxy. While the localisation will not be particularly good in this mode, by choosing the appropriate pointing locations for the telescopes it will be possible to use our multi-beam localisation techniques [50] to get positions to a few arcminutes. Compared with the ATA Fly's Eye experiment, our proposed experiment will be more than an order of magnitude more sensitive while also covering a significant fraction of the sky.

Deep-Searches-of-Known-FRB-Locations Mode: Alongside the sheer increase in the number of 
FRBs detected the other major development is that FRB 121102 has exhibited repeated bursts seen both at Arecibo and with the GBT [51]. These bursts are characterised by a range of flux density and strongly varying spectral indices and a wide range of modulation indices that have so far prevented the detection of any possible underlying periodicity. The repeating nature indicates that they are not from a cataclysmic event and suggest that at least some FRBs are possibly associated with neutron star origins, like magnetars or giant pulses from radio pulsars. Crucial to understanding what this means for the FRB population as a whole is determining if all FRBs repeat or whether there are multiple classes. It is also essential to be able to localise these bursts accurately to be able to see if they are associated with galaxies, and where possible determine where in the galaxies they occur. Using 200 hours of observing time to follow up known FRBs at the MeerKAT sensitivity would allow us to place stronger limits on any repeat bursts while forming images from the transient buffer data will allow us to get better localisations for any repeat bursts. Two hundred hours allows one to follow up a significant sample of 20 sources each for 10 hours, which will provide crucially strong limits on any underlying pulse energy distribution. The follow up observations will typically be an hour in duration and distributed logarithmically in time to sample as many timescales as possible. Working with ThunderKAT would also allow one to use the simultaneous imaging data to understand the radio properties of any host.

\subsection{Follow Up Timing:}

After the initial discovery of a pulsar, most of the science can only be extracted by follow-up timing. Therefore, it is an absolutely crucial aspect of characterising the new TRAPUM pulsars to get a timing solution. In its most basic form this means getting an accurate position, period and period derivative so that one can compare the pulsar properties with the known pulsar population, and in particular for those sources that are found in our targeted searches we are interested in knowing their characteristic ages and their spin-down energies to compare with the SNRs and high energy emission, for example. We are also interested in determining whether or not the sources are potentially high precision timers and so useful for gravitational wave searches, and/or members of binary systems and so potentially useful for mass determinations or tests of gravity.

One of the crucial advantages of finding pulsars with an interferometer compared to single dishes, is the narrow beam of the tied-array beam it is discovered in, which means that one already starts with a precise position. This removes a large degree of the ambiguity that usually exists between the positional uncertainty and the period-derivative and so one need not have as many follow up observations in order to obtain a coherent timing solution [52]. Our experience with LOFAR has shown that even when the pulsar is found in an incoherent beam, we can use the tied-array beams in follow-up observations to give an accurate position for subsequent observations. We note that for binary pulsars, more frequent observations may be needed to determine the binary parameters. Timing of the MSPs requires the pulsar timing beams and hardware, however for the slower pulsars it will be possible to use the pulsar search beams (that is we don't need coherent dedispersion) that we are providing and thus allowing us to potentially time a number of pulsars simultaneously. We find that for an estimated total of $\sim 800-1000$ pulsars discovered by TRAPUM, across all the searches proposed here, of which approximately $10 \%$ are expected to be in binaries, we find that a total of 500 hours is required for initial follow up timing observations. 


\section{Achieving the proposed science goals}

We outline below the details of the approach we will implement to achieve these science goals.

\subsection{The 400-beam beamformer and real-time/offline processing resources}

Funds obtained by the MPIfR internally and from the Max-Planck Society will enable the implementation of S-band receivers (see elsewhere in these proceedings for more details) to, amongst other things, enable deep pulsar searches in the Galactic plane and Galactic centre. Combined with funds obtained by Stappers through an European Research Council Advanced Grant for the MeerTRAP project (see elsewhere in these proceedings), also support the development of a 400-beam tied-array beamformer and computing resources for the storage of long/deep, observations of the targets proposed here, for off-line acceleration processing. The MeerTRAP funding enables a realtime search for pulsars and fast transients in parallel and the implementation of a transient buffer which can store thirty seconds of data from each of the MeerKAT dishes to be used for localising transients and the better characterisation of the dispersion, width and polarisation characteristics of the transients. The addition of the 400-beam beamformer and offline processing enables the ability to perform the Galactic plane search in reasonable observing time and also enables the crucial acceleartion searches that will be used for the plane and globular cluster searches.

\subsection{Observing strategy and time request:}

Given the various observing modes, we have highlighted the observing strategy in detail for each individual science goal above. In general all observations will use the combination of the 400 tied-array beams and the incoherent beams when undertaking pulsar and fast transient searches. Moreover there will be a real-time search undertaken using the available processing hardware and then more detailed searches, offline, using the temporary storage capacity and extra computing capability. The size of this storage buffer will allow about 24 hours of observation to be stored and then require 2-3 days to process and so that will place some scheduling restrictions on when the next pulsar search observations can take place. In general there is no restriction on the time of day when pulsar search observations take place, but to take advantage of optical follow up of any transients that are found some night time would be useful. The exception is the Fly's Eye mode where we will use the 64 dishes independently and so they appear as if they are 64 different beams. Thus the processing requirements are reduced here and all processing will be done in real time. Also, for the Fly's Eye observations the majority of the observing time would preferably be at night to allow for the possibility for simultaneous optical detection. As with the discussion of the observing strategy, the observing time request is discussed in detail for each individual science area. This does not include calibration or slewing time, the latter is small (much less than 1\%) as our integration times are long, and we typically have many nearby pointings. Our experience with the WSRT and the VLA have shown that to phase up the array for beam-forming typically requires about 30 minutes of observing time for a $24 \mathrm{~h}$ run, so assuming scheduling blocks of that duration the calibration overhead is about $2-3 \%$. 


\subsection{Plan for creating and archiving the scientific data product:}

The data processing challenges associated with TRAPUM will build on the development of pulsar and fast transient search pipelines for surveys like HTRU-N/S, SUPERB, PALFA, LOTAAS and the SKA. In all observations apart from Fly's Eye we will be operating with 400 tied-array beams each with 4096 frequency channels, digitised to 8-bits and sampled every $64 \mu \mathrm{s}$, leading to a total data rate of 24 GByte/s. These data will be analysed in three different ways: Realtime commensal searching for fast radio transients and pulsars and offline processing to search for pulsars in binary systems. The real-time and offline analysis will use the hundreds of GPUs and $\sim 2$ PetaBytes of storage acquired through the MPIfR/MeerTRAP grants. Up to $24 \mathrm{~h}$ of data will be recorded for offline processing which will take place while the telescope is pursuing other projects.

This pulsar search raw data product is too large to be archived (i.e. in total more than 250 Petabytes), however we will keep a version with reduced frequency and time resolution (100th of the data volume) for potential reprocessing and confirmations. The main data product will be sets of pulsar candidates, including folded and dedispersed data cubes, and associated metadata. We will use our state-of-the art machine learning algorithms (e.g. [53]) to filter out the most likely pulsars and fast transients. All candidates will be stored, as they are sufficiently small volume, so that they can be re-analysed with new machine learning algorithms. We have a definition and description of these data products that we will implement and we have funding for long term archive of 2.5 PetaBytes.

The transient search processing will be undertaken at the same time as the real-time pulsar search. The added challenge of the transient pipeline is that we will need to generate triggers in real-time and output the contents of the transient buffers and trigger MeerKAT and other facilities as quickly as possible. The mechanics of this process will be built on those established by ThunderKAT/4piSky. The output data products will consist mainly of candidates, which will also be stored for further assessment with improved algorithms. For those detections deemed to be sufficiently interesting a piece of the time-frequency-polarisation data cube surrounding the transient epoch will be added to the long term archive. The Fly's Eye mode observations will be processed very similarly to the real time transient detection mode, where in this case there will be 64 individual telescope inputs and so the data rates and processing requirements will be less than those for the tied-array searches. The output data products and archiving plans will be the same.

Triggering of the individual telescope transient buffers will result in data spanning the duration of the dispersed event, to be distributed to the data storage identified above. These data will be imaged in near real time at high time resolution. The transient will be identified using imaging transient pipelines like those developed for LOFAR/ThunderKAT. The image and tied-array beamformed data will be archived. Storing the raw telescope data long term will not be possible, but they will be kept for a few months.

\subsection{Commensal Observing:}

TRAPUM includes its own commensal observing, that is, it will undertake transient searches commensally on all its pulsar search and FRB follow up observations described above. The Fly's Eye mode observations require a very specific observing set up with the dishes pointed in many different directions and so they cannot be carried out in a commensal mode, but other projects, 
could potentially piggyback on these observations, although there will be no interferometric data products of use, except perhaps autocorrelations. All of the targeted and pointing observations could potentially be done in commensal mode if there were projects interested in imaging these sources, for example supernova remnants or external galaxies. However we do require access to the broad bandwidths with wide channelisation and consideration would have to be given to the noise source and calibration strategies, similarly we would need to make sure that the on source time, between observations of calibrators, matched the requirements for our periodic search routines. The same is also true of the Census regions, however here we would most likely want to decide the location of each pointing, but this could be negotiated. We note that it may also be possible to undertake some of the follow up pulsar timing observations in parallel with MeerTIME where they have overlapping regions of the sky and sufficient pulsar timing beams available and vice versa. The LMC/SMC survey could be carried out commensally with any future open time proposal to study these regions. We are also collaborating strongly with ThunderKAT on imaging and beamformed observations of FRBs.

\section{Conclusions}

TRAPUM will use MeerKAT to revolutionise our understanding of pulsars and fast transients and enable their use to test physical theories. We thank all of the TRAPUM team members for their contributions to the text in this proceedings.

\section{References}

[1] A. A. Abdo, et al., The Second Fermi Large Area Telescope Catalog of Gamma-Ray Pulsars, ApJS 208, p. 17 (2013).

[2] P. M. Saz Parkinson, et al., Classification and Ranking of Fermi LAT Gamma-ray Sources from the 3FGL Catalog using Machine Learning Techniques, ApJ 820, p. 8 2016).

[3] J. W. T. Hessels, et al., PSR J1856+0245: Arecibo Discovery of a Young, Energetic Pulsar Coincident with the TeV $\gamma$-Ray Source HESS J1857+026, ApJ 682, L41 (2008).

[4] H. T. Cromartie, et al., Six New Millisecond Pulsars from Arecibo Searches of Fermi Gamma-Ray Sources, ApJ 819, 34 (2016).

[5] J. Hessels, et al., Pulsars in Globular Clusters with the SKA, Advancing Astrophysics with the Square Kilometre Array (AASKA14) , 47 (2015).

[6] P. J. Turk and D. R. Lorimer, An empirical Bayesian analysis applied to the globular cluster pulsar population, MNRAS 436, 3720 (2013).

[7] S. M. Ransom, et al., Twenty-One Millisecond Pulsars in Terzan 5 Using the Green Bank Telescope, Science 307, 892 (2005).

[8] F. Verbunt and P. C. C. Freire, On the disruption of pulsar and X-ray binar ies in globular clusters, A\&A 561, A11 (2014).

[9] R. S. Lynch, D. R. Lorimer, S. M. Ransom and J. Boyles, A Population of Non-recycled Pulsars Originating in Globular Clusters, ApJ 756, 78 (2012). 
[10] P. C. C. Freire, S. M. Ransom and Y. Gupta, Timing the Eccentric Binary Millisecond Pulsar in NGC 1851, ApJ 662, 1177 (2007).

[11] M. E. DeCesar, et al., A Highly Eccentric 3.9 Millisecond Binary Pulsar in the Globular Cluster NGC 6652, ApJ 807, L23 (2015).

[12] J. W. T. Hessels, et al., A 1.4-GHz Arecibo survey for pulsars in globular clusters, ApJ 670, 363 (2007).

[13] J. P. Ridley, et al., Eight new radio pulsars in the Large Magellanic Cloud, MNRAS 433, 138 (2013).

[14] L. G. Spitler, et al., A repeating fast radio burst, Nature 531, 202 (March 2016).

[15] M. A. McLaughlin and J. M. Cordes, Searches for giant pulses from extragalactic pulsars, ApJ 596, 982 (2003).

[16] M. J. Keith, et al., The High Time Resolution Universe Pulsar Survey - I. System configuration and initial discoveries, MNRAS 409, 619 (2010).

[17] J. H. Taylor and J. M. Weisberg, Further experimental tests of relativistic gravity using the binary pulsar PSR 1913+16, ApJ 345, 434 (1989).

[18] M. Kramer, et al., Tests of General Relativity from Timing the Double Pulsar, Science 314, 97 (2006).

[19] N. Wex and S. Kopeikin, Frame dragging and other precessional effects in black hole-pulsar binaries, ApJ 513, 388 (1999).

[20] K. Liu, et al., Prospects for Probing the Spacetime of Sgr A* with Pulsars, ApJ 747, p. 1 (2012).

[21] R. Eatough, et al., Observing Radio Pulsars in the Galactic Centre with the Square Kilometre Array, Advancing Astrophysics with the Square Kilometre Array (AASKA14), 45 (2015).

[22] C.-A. Faucher-Giguère and A. Loeb, Pulsar-black hole binaries in the Galactic Centre, MNRAS 415, 3951 (2011).

[23] D. Psaltis, N. Wex and M. Kramer, A Quantitative Test of the No-hair Theorem with Sgr A* Using Stars, Pulsars, and the Event Horizon Telescope, ApJ 818, 121 (2016).

[24] R. P. Eatough, et al., A strong magnetic field around the supermassive black hole at the centre of the Galaxy, nat 501, 391 (2013).

[25] L. Goodenough and D. Hooper, Possible Evidence For Dark Matter Annihilation In The Inner Milky Way From The Fermi Gamma Ray Space Telescope, ArXiv e-prints 0910.2998 (2009).

[26] T. D. Brandt and B. Kocsis, Disrupted Globular Clusters Can Explain the Galactic Center Gamma-Ray Excess, ApJ 812, 15 (2015).

[27] J. Bramante and T. Linden, Detecting Dark Matter with Imploding Pulsars in the Galactic Center, Physical Review Letters 113,. 191301 (2014).

[28] J. P. Macquart, et al., Fast Transients at Cosmological Distances with the SKA, Advancing Astrophysics with the Square Kilometre Array (AASKA14), 55 (2015).

[29] F. Calore, et al., Radio Detection Prospects for a Bulge Population of Millisecond Pulsars as Suggested by Fermi-LAT Observations of the Inner Galaxy, ApJ 827, 143 (2016).

[30] L. G. Spitler, et al., Pulse Broadening Measurements from the Galactic Center Pulsar J1745-2900, ApJl 780, L3 (2014). 
[31] S. D. Bates, D. R. Lorimer, A. Rane and J. Swiggum, PSRPOPPy: an open-source package for pulsar population simulations, MNRAS 439, 2893 (2014).

[32] C. Ng, et al., The High Time Resolution Universe Pulsar Survey - XII. Galactic plane acceleration search and the discovery of 60 pulsars, MNRAS 450, 2922 (2015).

[33] D. Thornton, et al., A Population of Fast Radio Bursts at Cosmological Distances, Science 341, 53 (2013).

[34] M. A. McLaughlin, et al., Transient radio bursts from rotating neutron stars, Nature 439, 817 (2006).

[35] J. S. Deneva, et al., Arecibo Pulsar Survey Using ALFA: Probing Radio Pulsar Intermittency And Transients, ApJ 703, 2259 (2009).

[36] E. F. Keane and M. Kramer, On the birthrates of Galactic neutron stars, MNRAS 391, 2009 (2008).

[37] D. R. Lorimer, et al., A Bright Millisecond Radio Burst of Extragalactic Origin, Science 318, 777 (2007).

[38] L. G. Spitler, et al., Fast Radio Burst Discovered in the Arecibo Pulsar ALFA Survey, ApJ 790, 101 (2014).

[39] K. Masui, et al., Dense magnetized plasma associated with a fast radio burst, Nature 528, 523 (2015).

[40] D. Hutsemékers and H. Lamy, Confirmation of the existence of coherent orientations of quasar polarization vectors on cosmological scales, $A \& A$ 367, 381 (2001).

[41] M. J. Rees, A better way of searching for black-hole explosions, Nature 266, 333 (1977).

[42] H. Falcke and L. Rezzolla, Fast radio bursts: the last sign of supramassive neutron stars, $A \& A \mathbf{5 6 2}$, 137 (2014).

[43] E. F. Keane, et al., The host galaxy of a fast radio burst, Nature 530, 453 (2016).

[44] A. Loeb, Y. Shvartzvald and D. Maoz, Fast radio bursts may originate from nearby flaring stars, MNRAS 439, L46 (2014).

[45] S. R. Kulkarni, et al., Giant Sparks at Cosmological Distances?, ApJ 797, 70 (2014).

[46] B. P. Abbott, et al., Astrophysical Implications of the Binary Black-hole Merger GW150914, ApJl 818, L22 (2016).

[47] C. J. Law, et al., A Millisecond Interferometric Search for Fast Radio Bursts with the Very Large Array, ApJ 807, p. 16 (July 2015).

[48] A. P. V. Siemion, et al., The Allen Telescope Array Fly’s Eye Survey for Fast Radio Transients, ApJ 744, 109 (2012).

[49] D. J. Champion, et al., Five new fast radio bursts from the HTRU high-latitude survey at Parkes: first evidence for two-component bursts, MNRAS 460, L30 (July 2016).

[50] M. Obrocka, B. Stappers and P. Wilkinson, Localising fast radio bursts and other transients using interferometric arrays, $A A$ 579, 69 (2015).

[51] P. Scholz, et al., The repeating Fast Radio Burst FRB 121102: Multi-wavelength observations and additional bursts, ApJ 833, 177 (2016).

[52] B. Bhattacharyya, et al., The GMRT High Resolution Southern Sky Survey for Pulsars and Transients. I. Survey Description and Initial Discoveries, ApJ 817, 130 (2016).

[53] R. J. Lyon, et al., Fifty Years of Pulsar Candidate Selection: From simple filters to a new principled real-time classification approach, MNRAS 459, 110 (2017). 